\title{
Localized Acoustic Surface Modes
}

\author{
Mohamed Farhat ${ }^{1, *}$, Pai-Yen Chen ${ }^{2}$, and Hakan Bağcı ${ }^{1}$ \\ ${ }^{1}$ Division of Computer, Electrical, and Mathematical Sciences and Engineering, \\ King Abdullah University of Science and Technology (KAUST) \\ Thuwal 23955-69100, Saudi Arabia \\ ${ }^{2}$ Department of Electrical and Computer Engineering, Wayne State University, \\ Detroit, Michigan 48202, USA \\ *corresponding author, E-mail: mohamed. farhatekaust.edu.sa
}

\begin{abstract}
We introduce the concept of localized acoustic surface modes (ASMs). We demonstrate that they are induced on a two-dimensional cylindrical rigid surface with subwavelength corrugations under excitation by an incident acoustic plane wave. Our results show that the corrugated rigid surface is acoustically equivalent to a cylindrical scatterer with uniform mass density that can be represented using a Drude-like model. This, indeed, suggests that plasmonic-like acoustic materials can be engineered with potential applications in various areas including sensing, imaging, and cloaking.
\end{abstract}

\section{Introduction}

The current interest in plasmonics [1,2] is fueled by its promising applications in various areas [3] ranging from Biosensing [4], subwavelength imaging [5], energy harvesting [6], nanoelectronics [7], lighting [8], and photovoltaics [9]. Plasmonics studies the interaction of electromagnetic fields with metals at optical frequencies [2], and more recently, with graphene layers at terahertz (THz) frequencies [10-13]. This interaction results in generation of surface plasmon polaritons, i.e. strong electromagnetic fields localized to a subwavelength region on the interface between the metal and dielectric [2]. Field localization and amplification take place at frequencies around the plasma frequency $\omega_{p}$ of the metal used. For example, for gold, $\omega_{p}=2 \pi \times 350 \mathrm{THz}$, which explains why surface plasmons are induced on metals only at optical and near-infrared frequencies [14]. To overcome this limitation, Pendry et al. have proposed to use a corrugated metal surface patterned with subwavelength holes or slits, to generate surface plasmons at $\mathrm{THz}$ and gigahertz $(\mathrm{GHz})$ frequencies, [15] and showed that the effective permittivity of the equivalent medium possesses a plasma-like frequency, which can be tuned by varying the geometrical parameters of the corrugated surface [15]. These engineered surface plasmons, termed "spoof" plasmons, have opened new vistas in designing plasmonic devices/metamaterials with operating frequencies at $\mathrm{THz}$ and $\mathrm{GHz}$ parts of the spectrum [16-22].

Similarly, there has been an increasing interest in designing acoustic metamaterials, since Sheng et al. have demonstrated, for the first time, that dynamic mass density and bulk modulus can be obtained using locally resonant sonic materials [23]. However, unlike photonic metamaterials, which make use of surface plasmons to generate the desired dispersion characteristics, acoustic metamaterials almost exclusively rely on geometrical properties of their meta-atoms. This is due to the fact that surface plasmons do not exist naturally in acoustics [24]. As a result, design of acoustic metamaterials has made use of mostly phononic (or sonic) crystals, counterpart of photonic crystals, and resonant cavities or pipes [24-35]. This is, from many aspects, a major limitation of acoustic metamaterials, and their applications in areas that could benefit from acoustic plasmonic-like features.

To circumvent this limitation, one-dimensional (1-D) structures with subwavelength gratings, which can support acoustic modes confined to the surface, have been proposed [36-38]. It should be noted here that these modes are "acoustic versions" of the propagating electromagnetic surface plasmons and should not be confused with "acoustic surface plasmons" that correspond to collective lowfrequency excitations at metal surfaces [39,40]. Even though acoustic modes propagating on corrugated rigid surfaces have been well studied, to the best of our knowledge, their localized versions are yet to be investigated. To this end, in this work we introduce the concept of localized acoustic surface modes (ASMs) supported by corrugated rigid surfaces and demonstrate that such surfaces are equivalent to uniform scatterers with mass density that can be represented using a Drude-like model. These are achieved in two steps. First we demonstrate that propagating ASMs could be generated on 1-D corrugated rigid surfaces. Then, the results of 1-D propagating ASMs are used as a guide to design a 2-D corrugated rigid surface that supports localized ASMs.

Our findings demonstrate for the first time that localized plasmons can go acoustics. These findings will open up new vistas in designing acoustic devices with promising 
applications in many areas, including non-destructive sensing, subwavelength focusing and resolution, cloaking, and medical imaging.

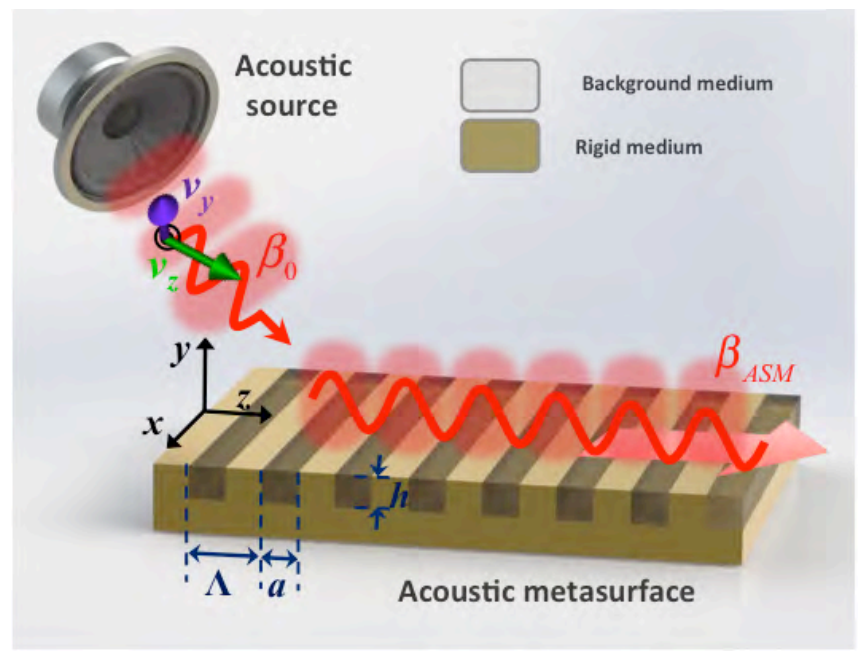

Figure1: 1-D rigid grating supporting propagating ASMs. An acoustic plane wave incident on the rigid grating induces propagating ASMs.

\section{Results}

In acoustics, a rigid material defines a medium where field propagation is prohibited. On surfaces of these materials, the condition of zero normal derivative of pressure, i.e. $\partial p / \partial n=0$, where $p$ is the pressure field and $\boldsymbol{n}$ is the surface normal, is satisfied. The same condition can be expressed, using the velocity field $\boldsymbol{v}$ as $\boldsymbol{n} \cdot \boldsymbol{v}=0$. These conditions ensure that pressure and velocity fields do not penetrate into the rigid obstacle. A good example of rigid materials is steel. The fields $p$ and $v$ satisfy the equation $i \omega p=\kappa \nabla \cdot v$, where $\kappa$ is the bulk modulus of the material, and $\omega$ is the frequency of excitation. This relation is valid for inviscid fluids, and is derived using the conservation of momentum assuming the absence of shear, and a time harmonic dependence $e^{-i \omega t}$ for the fields.

\subsection{Propagating ASMs induced on 1-D rigid gratings}

Consider a 1-D perforated acoustically rigid material as shown in Fig. 1. The width and the height of the air-filled periodic grooves are represented with $a$ and $h$, respectively. The period of the grating is $\Lambda$. The structure is excited by an acoustic pressure plane wave generated by a transducer residing in air. It is assumed that $a<<\lambda_{0}$, $\Lambda<\lambda_{0}$, and $h<<\lambda_{0}$, where $\lambda_{0}=2 \pi c_{0} / \omega$ is the wavelength in air. Here, $c_{0}$ is the speed of sound in air and $\omega$ is the frequency of the incident field. The grating in Fig. 1 supports propagating acoustic surface waves. The pressure and velocity fields associated with these waves can be expressed in the following form $[2,15]$

$$
\begin{aligned}
& p(y, z)=\frac{1}{\sqrt{\Lambda}} \frac{\beta_{0}}{\beta_{y}} e^{i \beta_{\mathrm{ASM}} z+i \beta_{y} y}, \\
& \boldsymbol{v}(y, z)=-\left(\beta_{\mathrm{ASM}} / \beta_{y} \hat{\mathbf{y}}+\hat{\mathbf{z}}\right) \frac{1}{\sqrt{\Lambda}} e^{i \beta_{\mathrm{ASM} z}+i \beta_{y} y} .
\end{aligned}
$$

Here, $\beta_{\mathrm{ASM}}$ represents the propagation constant of these waves, $\beta_{0}=\omega / c_{0}=2 \pi / \lambda_{0}$ is the free space wave number, and $\beta_{y}=\sqrt{\beta_{0}^{2}-\beta_{\mathrm{ASM}}^{2}}$. Note that the condition $\beta_{\mathrm{ASM}}<\beta_{0}=\omega / c_{0}$ should be satisfied for the waves described by Eq. (1) to be propagating. To find the dispersion relation, i.e. to express $\beta_{\mathrm{ASM}}$ in terms of $\omega$, the following steps are followed: (i) The expressions of the fields inside the air-filled periodic grooves for different diffraction orders are obtained under the assumption $a<<\lambda_{0}$ and $\Lambda<<\lambda_{0}$. (ii) Continuity conditions of the normal components of the velocity and the pressure are applied to obtain the reflection coefficient $\zeta_{0}$ associated with the specular diffraction [2]:

$$
\zeta_{0}=-\frac{1+i \Sigma_{0}^{2} \tan \left(\beta_{0} h\right) \beta_{0} / \beta_{y}}{1-i \Sigma_{0}^{2} \tan \left(\beta_{0} h\right) \beta_{0} / \beta_{y}} .
$$

Here, $\Sigma_{0}=\sqrt{a / \Lambda} \operatorname{sinc}\left(\beta_{\mathrm{ASM}} a / 2\right)$ is the secular overlap integral (sinc is the cardinal sine function). Note that Eq. (2) is valid for $a<<\lambda_{0}$ and $\Lambda<<\lambda_{0}$. (iii) It is clear from Eq. (2), that for evanescent waves, i.e. $\beta_{\mathrm{ASM}}>\beta_{0}, \zeta_{0}$ has one pole corresponding to the dispersion relation of the ASM:

$\beta_{\mathrm{ASM}}=\frac{\omega}{c_{0}} \sqrt{1+\Sigma_{0}^{4} \tan ^{2}\left(\omega h / c_{0}\right)}$.

First, acoustic field interactions on the grating without the rigid ground layer (i.e. only alternating layers of rigid material and air) are characterized. For this grating, $\Lambda=1 \mathrm{~mm}, h=1.78 \Lambda$, and $a=0.11 \Lambda$. The transmittance and reflectance of this structure are computed using a finiteelement solver for $\lambda_{0}$ changing from $0.1 \Lambda$ to $7 \Lambda$ and are plotted versus the normalized wavelength $\lambda_{0} / \Lambda$ in Fig. 2(a). It can be seen that more than one peak with amplitudes reaching high values (almost 1 for the first order resonance with the highest wavelength) appears in the transmittance spectrum. The resonance wavelengths can be calculated using matching conditions of the effective impedance of the alternating layers with that of the background medium, i.e. air. These characteristics of the spectrum can be understood in the context of extraordinary transmission through subwavelength apertures analyzed for electromagnetic fields [41] as well as for acoustic fields [42,43]. It has been, in particular, shown that the surface acoustic modes, which enable the extraordinary transmission in acoustics, are fundamentally different from electromagnetic surface plasmons, which enable the extraordinary transmission in 
electromagnetics and optics, because the acoustic modes always hybridize with Fabry-Perot modes and are not truly surface modes [43]. However, if one adds a rigid material below the alternating layers (as shown in Fig. 1), it is possible to have ASMs similar to spoof plasmons of electromagnetics, which is the contribution of the work described here.
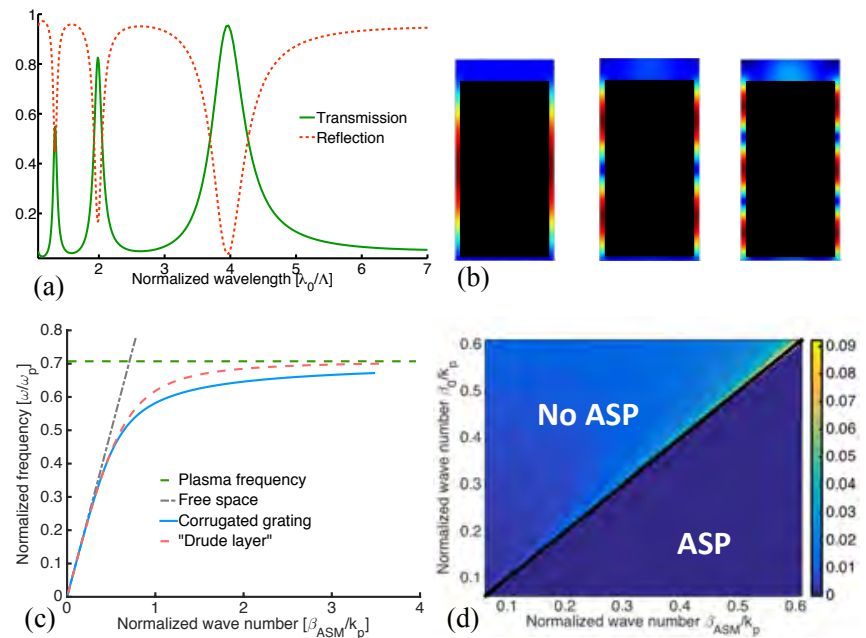

Figure2: Characterization of ASMs on the 1-D rigid grating. (a) Transmission/reflection spectrum of the alternating layers of the rigid material and air (without the ground layer made of rigid material) vs. the wavelength normalized with grating period. (b) Pressure field distribution around the rigid material layer at wavelengths corresponding to maxima of the transmittance (first, second and third order modes, respectively). (c) Dispersion curve associated with the propagating ASMs. (d) Phase of the transmittance $\zeta_{0}$ given by Eq. (2).

Next, the ASMs propagating on the grating with the rigid ground layer (as seen in Fig. 1) are characterized. The same values of $\Lambda, h$, and $a$ are used. Figure 2(c) plots the dispersion relation associated with the ASMs propagating on the grating, [described by Eq. (3)], versus the normalized wave number $\beta_{\mathrm{ASM}} / k_{p}$. Here, $k_{p}=\pi / 2 h$. In Fig. 2(c), also, the dispersion curve of ASMs propagating on the interface between air and a material, whose density is represented using the Drude-like model with plasma-like frequency $\omega_{p}=c_{0} \pi / \sqrt{2} h$, is plotted. The figure shows that for small values of the wave number (in the static limit), both dispersion relations are linear and identical to that in air (marked with black dotted line). When the wave number is increased, the dispersion curves get "flatter" and eventually converge to $\omega_{p} / \sqrt{2}$ (marked with green dotted line) outside the sound-cone (i.e. $\omega \geq c_{0} \beta_{\mathrm{ASM}}$ ). This behavior is very typical of electromagnetic surface plasmons observed at optical frequencies [2] and shows that the rigid grating gives rise to similar characteristics in acoustics. These results suggest that the rigid grating can be effectively treated as a homogeneous Drude-like layer with plasma-like frequency $\omega_{p}=c_{0} \pi / \sqrt{2} h$, which depends only on the speed of sound and the height of the grooves.

\subsection{Localized ASMs induced on corrugated 2-D surfaces}

Consider now an infinitely long corrugated cylindrical surface, made of acoustically rigid material, as shown in Fig. 3(a). The surface is modeled in 2-D space, as a corrugated circular curve residing on the $x-y$ plane. The radius of the curve is $R$, and it is corrugated into $N$ sectors, of equal arc length $R_{N}=2 \pi R / N$. The complementary corrugation [shown in gray in Fig. 3(a)] is filled with a material with density $\rho_{r} \rho_{0}$ and modulus $\kappa_{r} \kappa_{0}$. Here, $\rho_{0}=1.225 \mathrm{~kg} / \mathrm{m}^{3}$ and $\kappa_{0}=1.4 \times 10^{5} \mathrm{~Pa}$, are the density and modulus of air, where the structure is residing. The corrugated cylinder is excited by an acoustic plane wave, propagating in the $x$ direction, with unit amplitude pressure field and equal velocity field components in $x$ and $y$ directions. It is assumed that $R_{N}<<\lambda_{0}$.

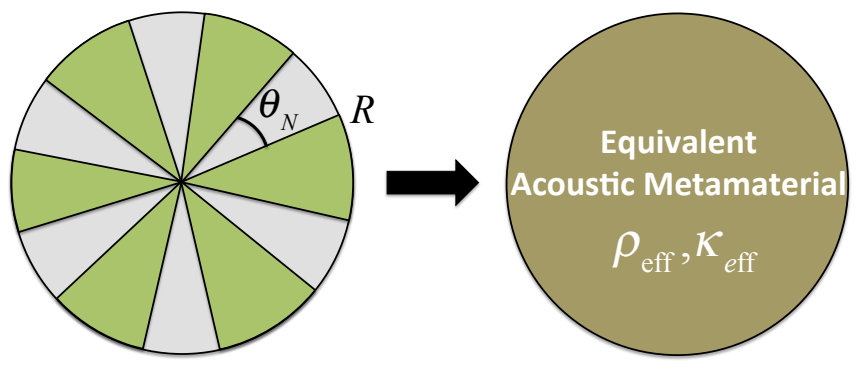

Figure3: 2-D Corrugated rigid cylinder and its equivalent model. (a) Corrugated rigid cylindrical surface of radius $R$ with $N$ sectors. (b) The complete circular region that is acoustically equivalent to the corrugated surface in (a). Effective mass density and bulk modulus of this region, $\rho_{\text {eff }}$ and $\kappa_{\text {eff }}$, depend on the geometry of the corrugated surface and the properties of the material filling the complementary corrugation.

The simulations of acoustic interactions on the corrugated cylinder are carried out using a finite-element solver, for $\lambda_{0}$ changing from $2.5 R$ to $5 R$. For these simulations, $\quad R=100 \mathrm{~mm}$ and $N=120 ;$ and the complementary corrugation is filled with air, i.e. $\rho_{r}=1$ and $\kappa_{r}=1$. After each simulation, the scattering cross section, $\sigma_{\mathrm{SCS}}$, of the corrugated cylinder is computed (see Methods section). Note that since there is no absorption (i.e. loss) associated with the rigid surface, $\sigma_{\mathrm{SCS}}$ is equal to the extinction cross section. Figure 4 (a) plots $\sigma_{\mathrm{SCS}} / 2 R$ of the corrugated cylinder vs. $\lambda_{0} / R$. Several resonance peaks corresponding to the different resonances of the corrugated cylinder are identified. Similar to the 1-D grating scenario, this suggests that this structure is acoustically equivalent to a complete circular scatterer of radius $R$ with plasmoniclike effective medium properties [Fig. 3(b)]. Here, we propose that the effective density $\rho_{\text {eff }}$ of this medium can 
be represented in terms of a Drude-like model, as was done for the 1-D case in the previous section:

$\rho_{\mathrm{eff}}(\omega) / \rho_{0}=1-\omega_{p}^{2} / \omega^{2}$

It is assumed that the effective bulk modulus of the effective medium $\kappa_{\text {eff }}=\kappa_{0}$. It should be clear here that the plasma-like frequency $\omega_{p}$ depends on the geometry of the corrugated cylinder, defined by $R$ and $\theta_{N}$, and the properties of the material filling the complementary corrugation, defined by $\rho_{r}$ and $\kappa_{r}$. For frequencies $\omega<\omega_{p}$, one can see immediately that the density takes negative values, contrary to all existing materials in nature. This means that the acceleration becomes $\pi$ out of phase with the dynamic pressure gradient. Electromagnetic equivalent of this phenomena is having $\operatorname{Re}\left(\varepsilon_{r}\right)<0$, where $\varepsilon_{r}$ is the relative dielectric permittivity. Noble metals satisfy this condition at optical frequencies and have been extensively used in the generation of plasmonic effects. The potential of having materials with density described with Eq. (4) in acoustics [as seen in Fig. 4(a)], even in the effective long wavelength limit, would be a tremendous breakthrough by no less than making most of the applications of plasmonics adapted to acoustics.

To verify these claims, $\sigma_{\mathrm{SCS}}$ of the equivalent complete circular scatterer of radius $R$ filled with effective medium with $\rho_{\text {eff }}$ and $\kappa_{\text {eff }}$ is compared to that of the corrugated cylinder. Finite-element simulations are carried out using the same excitation parameters. For these simulations, the plasma-like frequency of the effective medium is $\omega_{p}=c_{0} \pi / \sqrt{2} R$, which depends clearly, only on the radius of the corrugated cylinder, and as stressed above, only valid in the limit when the size of the building sectors is much smaller than the wavelength. Note that this expression is predicted using the result of the 1-D grating analysis and it is same as the one provided in the previous section, by changing $h$ to $R$. For $R=100 \mathrm{~mm}$, plasma-like frequency becomes $\omega_{p}=2 \pi \times 1.2 \mathrm{kHz}$. Figure 4(a) compares $\sigma_{\mathrm{SCS}}$ of the corrugated cylinder and the equivalent model. One can see that both $\sigma_{\mathrm{SCS}}$ undergo multiple resonances in the range of wavelengths between $2.75 R$ and $3.5 R$. From this graph, one can see that the effective material approximation is quite accurate in predicting the spectral shapes of the resonances: A broadband first order mode at the largest wavelength, and narrowband higher order modes at smaller wavelengths can be observed for both scenarios. Additionally, the resonance wavelengths of the different modes can be fairly predicted using the equivalent scatterer. The discrepancy between $\sigma_{\mathrm{SCS}}$ of the corrugated cylinder and its equivalent model is due to the fact that the expression of $\omega_{p}$ is predicted from the analysis of a 1-D rigid grating, which may not be very accurate for the 2-D corrugated cylinder. To get more insight into the nature of the resonances seen in Fig. 4(a), upper and lower panels in
Fig. 4(b) plot the amplitude of the pressure field distribution around the resonance wavelengths of the modes for the corrugated cylinder and its equivalent model, respectively. Note that the scales of both panels are different, because for the corrugated case, the amplitude of the pressure field reaches very high values in the narrow space between the different sectors. These figures clearly show that the distribution of the pressure fields excited on the corrugated cylinder and its equivalent model are similar, as expected. The number of maxima of the modes of orders 6,8 and 10 (from left to right in the figure) is the same for both structures. These confirm the accuracy of "replacing" the corrugated cylinder by its equivalent model with dispersive mass density. Additionally, the peaks in the spectra of $\sigma_{\mathrm{SCS}}$ around $\lambda_{0}=3 R$ (i.e. $\omega=2 \pi c_{0} / 3 R$ ) are generated by the equivalent model, with negative mass density [see Eq. (4), note that $\left.2 \pi c_{0} / 3 R<\omega_{p}=c_{0} \pi / \sqrt{2} R\right]$. This just means that the corrugated cylinder behaves effectively as a negativedensity medium in this spectral range.
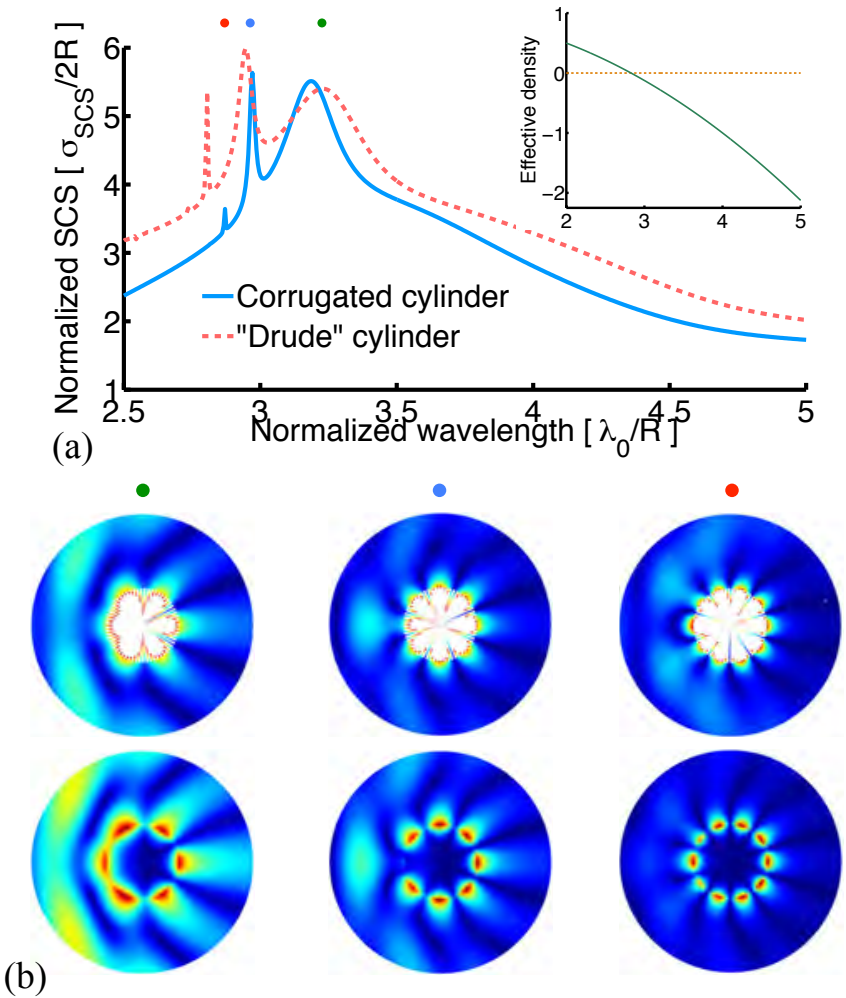

Figure 4: Comparison of acoustic field interactions on the corrugated cylinder and its equivalent model. (a) Scattering cross section of the corrugated cylinder and its equivalent model vs. the wavelength normalized with the radius of the cylinder. The inset shows the Drude-like density dispersion of the equivalent cylinder given by Eq. (4). (b) Pressure field distribution around the corrugated cylinder (upper three) and its equivalent model (lower three) at resonant frequencies of the three modes, marked with points of green, blue and red color respectively, in (a).

The equivalency between the acoustic responses of the rigid corrugated cylinder and the uniform cylinder with 
Drude-like density is only valid for acoustic fields external to the cylinders. The effective Drude cylinder cannot be accurately used to model the behavior of the acoustic fields inside the corrugations (sectors of the cylinder shown in Fig. 3). The explanation is that inside a Drude-like material, acoustic fields decay exponentially, whereas within the sectors of the corrugated rigid cylinder there exists a propagating acoustic mode leading to non-evanescent acoustic fields. To represent these fields inside the sectors using an effective material, one shall map the corrugated region into an effective anisotropic material, when the condition $2 \pi R / N<<\lambda_{0}$ is satisfied. Using a reasoning similar to that developed for TM electromagnetic waves [21], one can derive that $\rho_{r}=\lambda=2$ and $\rho_{\theta}=-\infty$. In addition, the results presented in Fig. 4 can be explained using a modal expansion technique, which provides an insight to the mechanism of the resonances. This technique relies on "matching" fields of the acoustic modes present outside the scatterer with those inside the sectors (or grooves) [44]. We start by expanding the fields in terms of Hankel functions of the first and second kinds (due to the symmetry of the problem):

$$
\begin{aligned}
& p_{0}(r, \theta)=\sum_{l=-\infty}^{+\infty} c_{l} H_{l}^{(1)}\left(\beta_{0} r\right) e^{i l \theta}, \\
& p_{1}(r, \theta)=d_{+} H_{0}^{(1)}\left(\beta_{0} r\right)+d_{-} H_{0}^{(2)}\left(\beta_{0} r\right) .
\end{aligned}
$$

Here, the subscripts 1 and 2 refer to regions external to the scatterer and the interior of the grooves, respectively. Applying the matching boundary conditions on the pressure and normal component of the velocity, we obtain the following resonance condition

$$
J_{1}\left(\beta_{0} R\right) H_{l}^{(1)}\left(\beta_{0} R\right)+2 J_{0}\left(\beta_{0} R\right) H_{l}^{(1)^{\prime}}\left(\beta_{0} R\right)=0,
$$

where $J_{0}(\cdot)$ and $J_{1}(\cdot)$ the Bessel functions of order zero and one, respectively. It should be noted here that this equation is obtained under the assumption that the dimensions of the grooves and the rigid sectors are the same. Three solutions (in terms of normalized wavelength) to Eq. (6), which correspond different multipole resonances of the corrugated cylinder, are marked with green, blue, and red circles on Fig. 4(a).

Physically, this means that each resonance that is observed in Fig. 4(a) corresponds to an integer number of wavelengths (of the acoustic mode) that fit into the perimeter of the cylinder [as seen in Fig. 4(b)]. This simple analytical model elucidates the geometrical origin of the localized ASMs and shows why the plasma-like frequency predicted from 1-D analysis should be related to the height of the grooves (here the radius of the cylinder). This also justifies the terminology of multipolar modes (dipole, quadrupole, etc.). Thus, it becomes clear from this picture that the acoustic modes associated with the grooves play the role of the collective oscillations of electrons in Drude metals, and is at the origin of (localized) ASMs.

\section{Discussion}

In this section, we further demonstrate that the characteristics of the localized ASMs induced on the corrugated cylinder can be tuned by changing the geometry of the corrugation and the material filling the complementary corrugation. Additionally, the possibility of using its plasmon-like resonant mode in acoustic sensing applications is discussed.

First, the effect of changing $\rho_{r}$, the relative mass density of the material filling in the complementary corrugation, on $\sigma_{\mathrm{SCS}}$ of the corrugated cylinder is characterized. For these simulations, the geometry parameters of the structure are kept the same while $\rho_{r}$ is varied from 0.8 to 2 . Figure $5(\mathrm{a})$ plots $\sigma_{\mathrm{SCS}}$ versus $\lambda_{0} / R$ for various values of $\rho_{r}$. A dramatic redshift in the resonant frequencies is observed as $\rho_{r}$ is increased. It should also be noted here that the spectral shape of $\sigma_{\mathrm{SCS}}$ is preserved. This is a behavior expected from a good sensor: a slight change in the medium (here represented by $\rho_{r}$ ) has a large effect on the observed properties of the sensor (here represented by $\left.\sigma_{\mathrm{SCS}}\right)$.
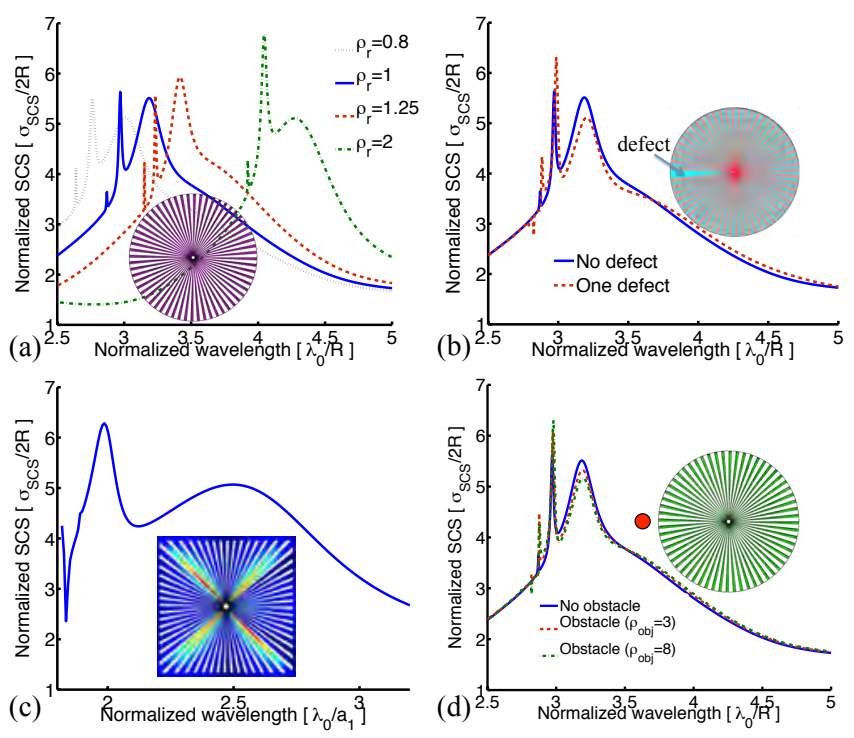

Figure 5: Comparison of acoustic field interactions on the corrugated cylinder and its equivalent model. (a) Scattering cross section of the corrugated cylinder and its equivalent model vs. the wavelength normalized with the radius of the cylinder. The inset shows the Drude-like density dispersion of the equivalent cylinder given by Eq. (4). (b) Pressure field distribution around the corrugated cylinder (upper three) and its equivalent model (lower three) at resonant frequencies of the three modes, marked with points of green, blue and red color respectively, in (a).

Next, the robustness of the structure to imperfections in the geometry of corrugation is investigated. For these simulations, one of the corrugation sections is made larger by increasing its angle from $2 \pi / N$ to $4 \pi / N$. The other geometry parameters are kept the same, and $\rho_{r}=1$ and 
$\kappa_{r}=1$. Figure 5(b) compares $\sigma_{\mathrm{SCS}}$ of the corrugated cylinder to that of the one with the modified corrugation. It is clear that $\sigma_{\mathrm{SCS}}$ is not affected that much; in particular the shape and the spectral location of the modes remain unchanged.

Next, the geometry changed from a corrugated circular cylinder to a corrugated square cylinder. For these simulations, the side of the square is $100 \mathrm{~mm}$ and the number of corrugation sectors $N=120 . \sigma_{\text {SCS }}$ of the square cylinder behaves in a similar way to that of the circular cylinder. The resonant frequencies of the modes can be identified, in Fig. 5(c), but they are observed at a wavelength range slightly different than those of the circular cylinder, similar to optical plasmons.

Last, a small circular scatterer with radius $r_{\mathrm{obj}}=0.1 R$ made out of from a material with mass density $\rho_{\text {obj }} \rho_{0}$ and bulk modulus $\kappa_{0}$ is placed in the vicinity of the corrugated cylinder. For these simulations, geometrical parameters of the cylinder are kept the same while $\rho_{\text {obj }}$ is varied between 3 and 8. Figure 5(d) compares $\sigma_{\text {SCS }}$ obtained in simulations without the scatterer, and with the scatterer with $\rho_{\text {obj }}=3$ and $\rho_{\mathrm{obj}}=8$. For the lowest order mode, the change in $\sigma_{\mathrm{SCS}}$ is not significant, whereas for the higher order modes, $\sigma_{\mathrm{SCS}}$ undergoes an abrupt change with increasing $\rho_{\mathrm{obj}}$. This suggests that the corrugated cylinder can be used in the detection of small objects with dimensions on the order of $\lambda_{0} / 30$.

We have introduced here the concept of localized ASMs. We have described in detail how they can be generated on rigid cylinders with subwavelength corrugations. Indeed our numerical experiments, which involve full wave simulations and analytical analysis, demonstrate that localized ASMs supported by such structures have characteristics that are very similar to those of the electromagnetic plasmons supported by metallic scatterers at optical frequencies. We envision that devices capable of supporting such acoustic modes might find interesting applications in biomedical sensing, imaging, cloaking, waveguiding, and enhancement of nonlinear acoustic field interactions.

\section{Methods: Computation of the SCS}

The scattering cross section $\sigma_{\mathrm{SCS}}$ is computed by integrating the acoustic energy flux density over a circular surface that is enclosing the scatterer:

$\sigma_{\mathrm{SCS}}=\frac{1}{2 \pi R_{0}} \oint_{S_{0}} \mathbf{P} \cdot \hat{\mathbf{n}} d s$.

Here, $S_{0}$ is the integration surface, $R_{0}$ is its radius, $\hat{\mathbf{n}}$ is the unit normal vector pointing outwards on $S_{0}$, and $\mathbf{P}$ is the time averaged acoustic energy flux density. Let $p$ and $\boldsymbol{v}$ denote pressure and velocity fields (in phasor domain) assuming time harmonic dependence $e^{-i \omega t}$, then,

$\overrightarrow{\mathbf{P}}=\operatorname{Re}\left(p e^{-i \omega t}\right) \cdot \operatorname{Re}\left(v e^{-i \omega t}\right)=\frac{1}{2} \operatorname{Re}\left(p v^{*}\right)+\frac{1}{2} \operatorname{Re}\left(p v e^{-2 i \omega t}\right)$,

is the instantaneous energy flux density. Here, “*” represents complex conjugation. Time averaging $\overrightarrow{\mathbf{P}}$ yields

$\mathbf{P}=\langle\overrightarrow{\mathbf{P}}\rangle=\frac{1}{T} \int_{T} \overrightarrow{\mathbf{P}} d t=\frac{1}{2} \operatorname{Re}\left(p \boldsymbol{v}^{*}\right)$,

where $T=2 \pi / \omega$ is the period of the oscillation in time. Inserting Eq. (9) into Eq. (7) yields $\sigma_{\mathrm{SCS}}$ in terms of an integral involving $p$ and $\hat{\mathbf{n}} \cdot \boldsymbol{v}$. For scatterers with arbitrary shapes, $p$ and $\boldsymbol{v}$ can only be computed using numerical tools. In this work, finite element method based commercial software COMSOL Multiphysics is used for this purpose: We use here the PDE module of COMSOL Multiphysics in the 2-D, to model the Helmholtz equation satisfied by the pressure field of acoustic waves, and we enforce Neumann boundary conditions to model rigid materials

\section{Acknowledgements}

This work was partially funded by King Abdulaziz City for Science and Technology's TIC (Technology Innovation Center) for Solid-state Lighting at KAUST.

\section{References}

[1] L. Novotny and B. Hecht, Principles of Nano-Optics (Cambridge University Press, Cambridge, 2006).

[2] S.A. Maier, Plasmonics: Fundamentals and Applications (Springer, New York, 2007).

[3] W.L. Barnes, A. Dereux, and T.W. Ebbesen, Nature 424, 824-830 (2003).

[4] J. Homola, S.S. Yee, and G. Gauglitz, Sens. Actuator BChem. 54, 3-15 (1999).

[5] D.K. Gramotnev and S.I. Bozhevolnyi, Nat. Photonics 4, 83-91 (2010).

[6] S.A. Maier, and H.A. Atwater, J. Appl. Phys. 98, 011101 (2005).

[7] N. Engheta, Science 317, 1698-1702 (2007).

[8] G. Lozano, et al., Light: Sci. Appl. 2, e66 (2013).

[9] H.A. Atwater and A. Polman, Nat. Mater. 9, 205-213 (2010).

[10] L. Ju, et al., Nat. Nanotech. 6, 630-634 (2011).

[11] M. Farhat, S. Guenneau, and H. Bağc1, Phys. Rev. Lett. 111, 237404 (2013).

[12] J. Schiefele, J. Pedros, F. Sols, F. Calle, and F. Guinea, Phys. Rev. Lett. 111, 237405 (2013).

[13] P.Y. Chen, M. Farhat, and A. Alù, Phys. Rev. Lett. 106, 105503 (2011). 
[14] G.V. Naik, V.M. Shalaev, and A. Boltasseva, Adv. Mater. 25, 3264-3294 (2013).

[15] J.B. Pendry, L. Martin-Moreno, and F.J. Garcia-Vidal, Science 305, 847-848 (2004).

[16] A.P. Hibbins, B.R. Evans, and J.R. Sambles, Science 308, 670-672 (2005).

[17]F.J. Garcia de Abajo and J.J. Saenz, Phys. Rev. Lett. 95, 233901 (2005).

[18]F.J. Garcia-Vidal, L. Martin-Moreno, and J.B. Pendry, J. Opt. A: Pure Appl. Opt. 7, 97-101 (2005).

[19] M.A. Kats, D. Woolf, R. Blanchard, N. Yu, and F. Capasso, Opt. Express 19, 14860-14870 (2011).

[20] R. Stanley, Nat. Photon. 6, 409-411 (2012).

[21] P.A. Huidobro, et al., Phys. Rev. X 4, 021003 (2014).

[22] A. Pors, E. Moreno, L. Martin-Moreno, J.B. Pendry, and F.J. Garcia-Vidal, Phys. Rev. Lett. 108, 223905 (2012).

[23] Z. Liu, et al., Science 289, 1734-1736 (2000).

[24]R.V. Craster and S. Guenneau, (eds.), Acoustic Metamaterials: Negative Refraction, Imaging, Lensing and Cloaking (Springer, Berlin, 2013).

[25] G. Goffaux, et al., Phys. Rev. Lett. 88, 225502 (2002).

[26] J. Li and C.T. Chan, Phys. Rev. E 70, 055602(R) (2004).

[27] S. Yang, et al., Phys. Rev. Lett. 93, 024301 (2004).

[28] J. Christensen, A.I. Fernandez-Dominguez, F. de LeonPerez, L. Martin-Moreno, and F.J. Garcia-Vidal, Nat. Phys. 3, 851-852 (2007).

[29] M. Farhat, S. Enoch, S. Guenneau, and A.B. Movchan, Phys. Rev. Lett. 101, 134501 (2008).

[30] M. Farhat, et al., Phys. Rev. E 77, 046308 (2008).

[31] S. Zhang, L. Yin, and N. Fang, Phys. Rev. Lett. 102, 194301 (2009).

[32] M. Farhat, S. Guenneau, and S. Enoch, EPL-Europhys. Lett. 91, 54003 (2010).

[33] P.Y. Chen, M. Farhat, S. Guenneau, S. Enoch, and A. Alù, Appl. Phys. Lett. 99, 191913 (2011).

[34] B.I. Popa, L. Zigoneanu, and S.A. Cummer, Phys. Rev. Lett. 106, 253901 (2011).

[35]I. Spiousas, D. Torrent, and J. Sanchez-Dehesa, Appl. Phys. Lett. 98, 244102 (2011).

[36]Z. He, et al., Phys. Rev. B 83, 132101 (2011).

[37] J. Christensen, Z. Liang, and M. Willatzen, Phys. Rev. B 88, 100301(R) (2013).

[38] J. Christensen, Z. Liang, and M. Willatzen, AIP Advances 4, 124301 (2014).

[39] B. Diaconescu, et al., Nature 448, 57-59 (2007).

[40] M. Smerieri, et al., Phys. Rev. Lett. 113, 186804 (2014).

[41] T.W. Ebbesen, H.J. Lezec, H.F. Ghaemi, T. Thio, and P.A. Wolff, Nature 391, 667-669 (1998).

[42] M.H. Lu, et al., Phys. Rev. Lett. 99, 174301 (2007).
[43] J. Christensen, L. Martin-Moreno, and F.J. GarciaVidal, Phys. Rev. Lett. 101, 014301 (2008).

[44] A.F. Harvey, IRE Trans. Microwave Theory Tech. 8, 30 (1960). 